\title{
An efficient and fast detection algorithm for multimode FBG sensing
}

\author{
Ganziy, Denis; Jespersen, O.; Rose, B.; Bang, Ole
}

\section{Published in:}

Proceedings of SPIE

Link to article, DOI:

$10.1117 / 12.2194305$

Publication date:

2015

Document Version

Publisher's PDF, also known as Version of record

Link back to DTU Orbit

Citation (APA):

Ganziy, D., Jespersen, O., Rose, B., \& Bang, O. (2015). An efficient and fast detection algorithm for multimode FBG sensing. In J. K. Hypolito, J. L. Fabris, \& W. J. Bock (Eds.), Proceedings of SPIE (Vol. 9634). [963445] SPIE - International Society for Optical Engineering. Proceedings of SPIE - The International Society for Optical Engineering https://doi.org/10.1117/12.2194305

\section{General rights}

Copyright and moral rights for the publications made accessible in the public portal are retained by the authors and/or other copyright owners and it is a condition of accessing publications that users recognise and abide by the legal requirements associated with these rights.

- Users may download and print one copy of any publication from the public portal for the purpose of private study or research.

- You may not further distribute the material or use it for any profit-making activity or commercial gain

- You may freely distribute the URL identifying the publication in the public portal 


\title{
An efficient and fast detection algorithm for multimode FBG sensing
}

\author{
D. Ganziy*a,, O. Jespersen ${ }^{\mathrm{a}}$, B. Rose ${ }^{\mathrm{a}}$, O. Bang ${ }^{\mathrm{b}}$ \\ ${ }^{a}$ Ibsen Photonics A/S, Ryttermarken 15-21, 3520 Farum, Denmark; \\ ${ }^{b}$ DTU Fotonik, Department of Photonics Engineering, Technical University of Denmark \\ 2800 Kgs. Lyngby, Denmark
}

\begin{abstract}
We propose a novel dynamic gate algorithm (DGA) for fast and accurate peak detection. The algorithm uses threshold determined detection window and Center of gravity algorithm with bias compensation. We analyze the wavelength fit resolution of the DGA for different values of signal to noise ratio and different typical peak shapes. Our simulations and experiments demonstrate that the DGA method is fast and robust with higher stability and accuracy compared to conventional algorithms. This makes it very attractive for future implementation in sensing systems especially based on multimode fiber Bragg gratings.
\end{abstract}

Keywords: wavelength detection, demodulation algorithm, fiber Bragg grating (FBG), fiber optic sensor

\section{INTRODUCTION}

Fiber Bragg gratings (FBGs) have become more and more attractive in recent years because they are small, costeffective, easy to manufacture, low weight, insensitive to electromagnetic interference and their optical spectra have good linear responses with respect to variations of temperature and strain [1]. It has been shown that polymer optical fibers (POF) can also be used in sensing systems with certain benefits $[2,3,4]$. The most common and commercially available FBG interrogation techniques are spectrometer based or swept laser based [5]. These techniques sample the reflected spectrum with a finite sample step, for spectrometers given by the pixel pitch in the diode array. The optical resolution of these techniques is often limited by the sample resolution and is relatively poor compared to, for example, Fabry-Perot filters [6] and Mach-Zehnder interferometers [7]. The resolution in the detected FBG peak position can be enhanced to sub-pixel level by applying different peak fitting algorithms, such as Center of Gravity (COG) [8] and Gaussian fitting [9]. However, the fitting algorithm should be chosen carefully to achieve the best wavelength fit resolution. Most of the conventional algorithms are designed to work with sharp Gaussian peaks and use a constant number of pixels for peak fitting. This can result in inaccurate results, when the peak shape is not sharp and narrow and if the peak shape changes during measurements.

Here we propose a fast and accurate peak detection algorithm, which is well suited for spectrometers with a limited number of pixels. The algorithm is based on a threshold-determined fitting window and a modified COG algorithm with bias compensation. Thus, the number of pixels used for peak determination is not constant and changes during measurements. This approach avoids sudden shift in the fitted wavelength and improves the wavelength fit resolution. Using simulations, we investigate the static and dynamic performance of the proposed method and compare it with four other algorithms: COG; least squares Gaussian fitting; linear phase operator (LPO) [10] and a fast phase correlation (FPC) algorithm [11].

\section{THE DYNAMIC GATE ALGORITHM (DGA) PRINCIPLE}

The basic principle of FBG sensing is to measure the reflected spectrum and to track the FBG peak position. Most conventional algorithms use a constant number of samples (pixels) for peak position calculations. The first step of these algorithms is to find the local maximum point and then take $n$ points (neighbors) to the left and $n$ points to the right of the maximum, so the total number of points is $2 n+1$. Problems with this approach may appear when there is uncertainty in the determination of the maximum point. For example, as shown in Fig. 1(a), the maximum can be point number 1, but due to noise, the maximum can jump to point number 2 . These jumps lead to changes in the points used for peak fitting. When point 1 is maximum, the selected points are between the two red dashed lines and when point 2 is

24th International Conference on Optical Fibre Sensors, edited by Hypolito José Kalinowski, José Luís Fabris, Wojtek J. Bock, Proc. of SPIE Vol. 9634, 963445 · C) 2015 SPIE CCC code: $0277-786 \mathrm{X} / 15 / \$ 18 \cdot$ doi: $10.1117 / 12.2194305$ 
maximum, the selected points are between the two blue dot-dashed lines. The sudden jumps in the fitting window may produce sudden shifts in the fitted wavelength as will be illustrated in Section 3.
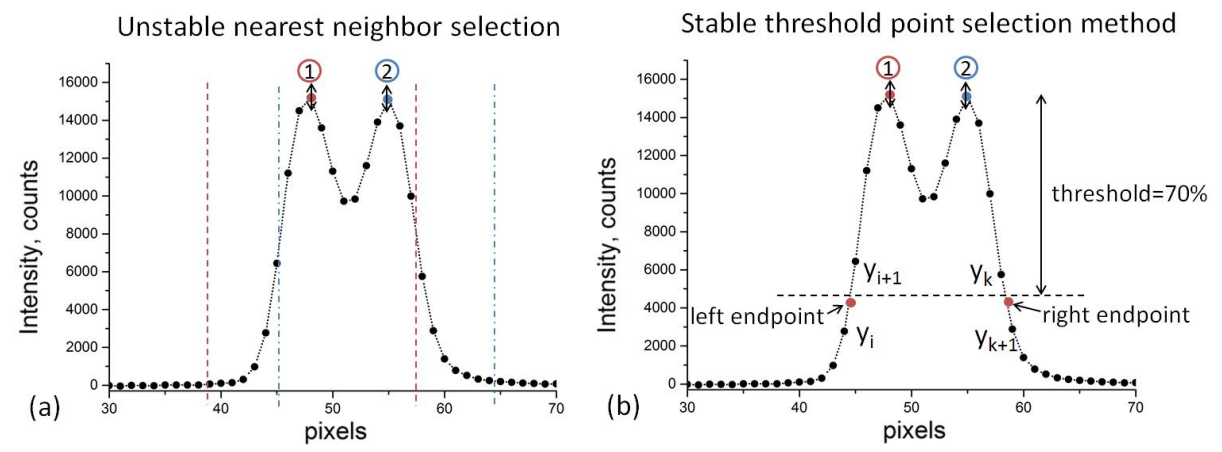

Figure 1. (a) The fitting window shift on double peak FBG spectrum, number of neighbors=9; (b) The threshold fitting window determination principle for a threshold of $=70 \%$ of the maximum

One way to avoid this problem is to simply increase the number of points in order to be sure to always cover the whole peak, however this approach has disadvantages: I) the fitting speed will be reduced; II) adding side points will increase the noise and decrease fit resolution; III) if peaks are close to each other the increased number of points may lead to the use of points from the neighboring peak. To overcome this problem we propose the threshold-based point selection, where all points higher than a threshold will be selected plus one left and one right endpoint, as shown in Figure 1(b). These endpoints are not part of the original data points, but are interpolated with the intensity and $\mathrm{x}$-coordinate of the left point $\left(y_{L}, x_{L}\right)$ and the right point $\left(y_{R}, x_{R}\right)$ found in the following way:

$$
y_{L}=T \frac{y_{i+1}^{2}-T^{2}}{y_{i+1}^{2}-y_{i}^{2}} ; \quad x_{L}=x_{i}+\frac{T-y_{i}}{y_{i+1}-y_{i}} ; \quad y_{R}=T \frac{y_{k}^{2}-T^{2}}{y_{k}^{2}-y_{k+1}^{2}} ; \quad x_{R}=x_{k}+\frac{y_{k}-T}{y_{k}-y_{k+1}} .
$$

Here $\left(y_{i}, x_{i}\right),\left(y_{i+1}, x_{i+1}\right),\left(y_{k}, x_{k}\right)$ and $\left(y_{k+1}, y_{k+1}\right)$ are the coordinates on each side of the threshold $T$ on the left and right side of the peak, respectively as illustrated in Fig. 1(b). The fitting window borders are limited by the left endpoint $x_{L}$ and by the right endpoint $x_{R}$ and it is no longer discrete. This fact allows us to avoid sudden jumps of the fitting window, which appears when the measured peak shifts. The last step of the proposed algorithm is to process the selected points. Originally, we selected the COG algorithm for this purpose, because it is fast and has high accuracy. But the COG is sensitive to the DC level of the measured signal, which may vary during measurements, thereby decreasing the accuracy. To overcome that problem we developed a modified COG algorithm with DC level compensation. The DC compensated COG, $C O G_{D C}$, of the measured spectrum between the point $x_{L}$ and $x_{R}$ is calculated in the following way:

$$
\begin{gathered}
C O G_{D C}=\frac{k c+i(c-r)}{k+c-r} \\
\text { with } c=\sum_{j=x_{L}}^{x_{R}} y_{j} x_{j} / \sum_{j=x_{L}}^{x_{R}} y_{j} ; \quad r=\sum_{j=x_{L}}^{x_{R}+1} y_{j} x_{j} / \sum_{j=x_{L}}^{x_{R}+1} y_{j} ; \quad k=\frac{2(i-r)+x_{R}-x_{L}+1}{2\left(x_{R}-x_{L}\right)} ; \quad i=\frac{x_{R}+x_{L}}{2} .
\end{gathered}
$$

The calculated wavelength shift $\lambda_{B}$ is equal to the DC compensated COG, where $x_{L}$ and $x_{R}$ are the left and the right interpolated points respectively, given by Eq. (1).

\section{SIMULATIONS AND RESULTS}

To evaluate the proposed algorithm, we performed simulations and comparisons using three different measured FBG spectra, as can be seen in Figure 2. In the first test we simulated the behavior of FBG 3 [see Fig 2 (c)] for an applied strain which is a linear function of time and with added white Gaussian noise with a signal to noise ratio (SNR) of $20 \mathrm{~dB}$. We selected 10 neighbors for the COG and Gaussian fitting, the DGA threshold was set at $70 \%$ of maximum. Figure 3 reports the calculated peak position as a function of time. The conventional algorithms demonstrate poor performance due to the sudden jumps in the fitting window caused by shifts in the maximum point determination, while the DGA shows a nice linear response. 

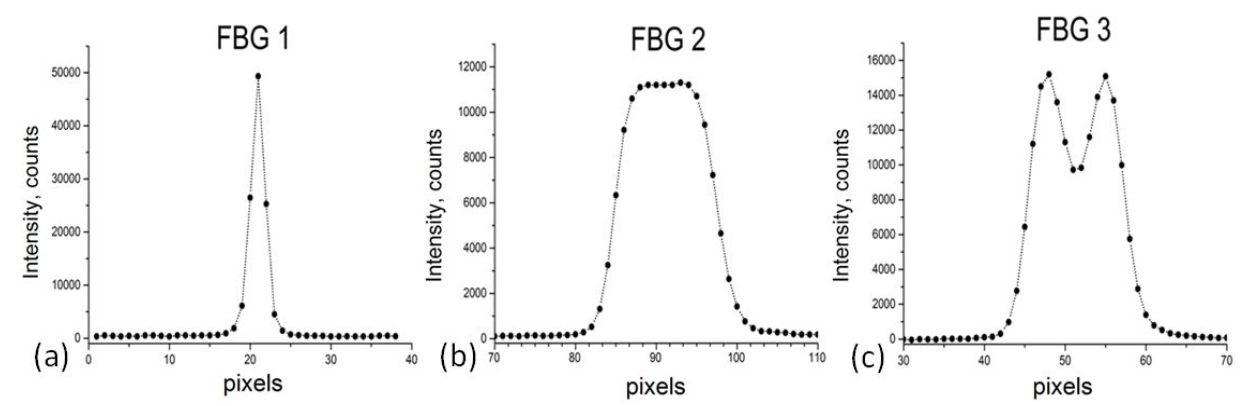

Figure 2. (a) FBG 1 -single mode spectrum, (b) FBG 2 and (c) FBG 3 - few mode spectra


Figure 3. Fitted center wavelength of FBG 3 calculated with the COG (a), Gaussian (b) and DGA (c) fitting

The aim of the second test was to calculate the wavelength fit resolution $\sigma$ given by Eq. (3).

$$
\sigma=\sqrt{\frac{1}{N} \sum_{i=1}^{N}\left(\lambda_{i}-\bar{\lambda}\right)^{2}}, N=100000
$$

We added white Gaussian noise with signal to noise ratios of 10 and $30 \mathrm{~dB}$ to the measured spectra (Fig. 2). For each SNR the peak position was calculated 100000 times to determine the wavelength fit resolution $\sigma$ [see Eq. 3]. For the first spectrum, FBG 1, which is a typical single mode FBG peak, the maximum point is stable. Therefore, there are no sudden jumps of the fitting window and all algorithms perform well, as can be seen in Figure 4. The number of neighbors $n$ was set to be 3 for FBG 1 .
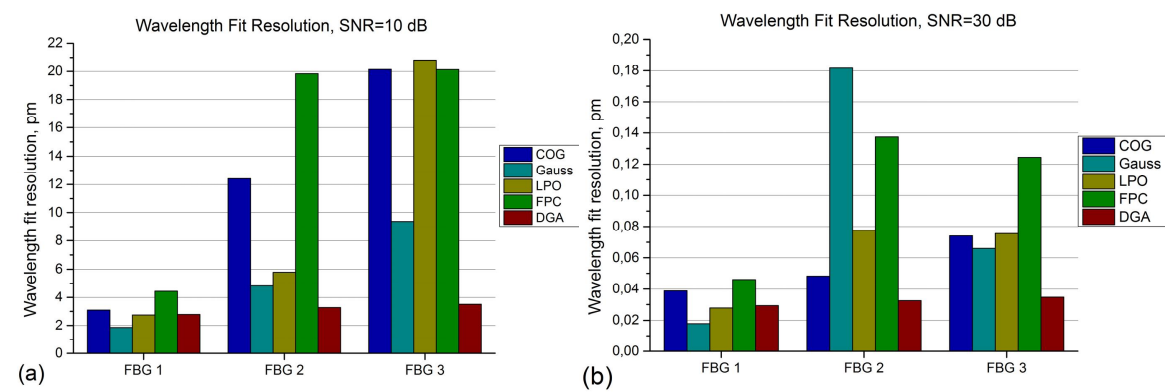

Figure 4. Wavelength fit resolution with low $\mathrm{SNR}=10 \mathrm{~dB}$ (a) and high $\mathrm{SNR}=30 \mathrm{~dB}$ (b).

The problems appear with FBG 2 and FBG 3, where the maximum position is not stable. In order to overcome the problem with the sudden jumps appearing when using the conventional algorithms [see Fig. $2(a, b)$ ], we increased the number of neighbors to cover the whole peak. We selected 12 and 14 nearest neighbors for FBG 2 and FBG 3, respectively. The threshold in the DGA was set at $70 \%$ for all measurements. As can be seen our algorithm shows the best fit resolution for FBG 2 and FBG 3 for both low and high SNR (Fig. 4). Compared to the best conventional algorithms, the DGA improves the fit resolution by $32 \%$ and 33\% for FBG 2 and $63 \%$ and $47 \%$ for FBG 3 for SNR=10 $\mathrm{dB}$ and $30 \mathrm{~dB}$, respectively. When the peak shape is known, as for example for FBG 1 with the Gaussian shape, the best fit resolution is obtained with the Gaussian fitting. However, for the FBG 1 the DGA improves the wavelength resolution by $24 \%$ and $37 \%$ compared to the COG and the FPC algorithms, respectively, and shows almost the same result as the LPO algorithm. 
The computation speed is the other important parameter for the performance evaluation of the proposed algorithm. It should be noted that the absolute computation speed depends on the number of points and the number of peaks. We measured the computation speed using FBG spectra shown in Figure 2 and then normalized it using the COG speed as a reference. The same data was fed to all algorithms, the number of neighbors and the threshold level was set to achieve the best fit resolution. All algorithms were implemented in LabVIEW. Table 1 reports the average relative speed for all different algorithms.

Table 1. Average relative speed of computation.

\begin{tabular}{|c|c|c|c|c|c|}
\hline Algorithm & COG & Gauss & LPO & FPC & DGA \\
\hline Relative speed, \% & 100 & 10 & 74 & 21 & 60 \\
\hline
\end{tabular}

The DGA is 6 times faster compared to the Gaussian fitting and only $40 \%$ slower than the simplest COG algorithm. The proposed method represents a good compromise between the fit resolution, robustness and computation speed. We have also carried out several experiments with different FBG sensors to evaluate the dynamic behavior and will demonstrate these results and results on the effectiveness of the method at the conference.

\section{CONCLUSION}

In this paper we presented an efficient and fast detection algorithm for multimode FBG sensing based on a threshold determined detection window and a bias compensated COG. The proposed algorithm demonstrated high robustness with highly improved wavelength fit resolution compared with conventional algorithms and the computation speed is 6 times faster than for Gaussian fitting algorithm. These properties make the DGA an attractive and suitable method for future implementation in sensing systems based on multimode fiber Bragg gratings.

\section{ACKNOWLEDGMENTS}

The research leading to these results has received funding from the People Programme (Marie Curie Actions) of the European Union's Seventh Framework Programme FP7/2007-2013/ under REA grant agreement n 608382.

\section{REFERENCES}

[1] Kersey, A. D., Davis, M. A., Patrick H. J., LeBlanc, M., Koo, K. P., Askins, C. G., Putnam, M. A. and Friebele, E.J., "Fiber grating sensors," J. Lightwave Technol., 15, 1442-1442 (1997).

[2] Xiong, Z., et al., "Highly tunable Bragg gratings in single-mode polymer optical fibers," Ieee Photonics Technology Lett., 11, 352-354 (1999).

[3] Yuan, W., Stefani, A., Bang, O., "Tunable polymer Fiber Bragg Grating (FBG) inscription: Fabrication of dualFBG temperature compensated polymer optical fiber strain sensors," IEEE Photon. Technol. Lett. 24, 401-403 (2012)

[4] Stefani, A., Andresen, S., Yuan, W., Herholdt-Rasmussen, N., Bang, O., "High Sensitivity Polymer Optical Fiber- Bragg-Grating-Based Accelerometer," IEEE Photon. Technol. Lett. 24, 763-765 (2012)

[5] Ball, G. A., Morey, W. W., and Cheo, R. K., "Fiber laser source/analyzer for Bragg grating sensor array interrogation," J. Lightwave Technol., 12, 700-703 (1994).

[6] Kersey, A. D., Berkoff, T. A., and Morey, W.W., "Multiplexed fiber Bragg grating strain-sensor system with a fiber Fabry-Perot wavelength filter," Optics Lett., 18, 1370-1372 (1993).

[7] Kersey, A. D., Berkoff, T. A., and Morey, W.W., "High-resolution fibre-grating based strain sensor with interferometric wavelength-shift detection," Electronics Lett., 28, 236-238 (1992).

[8] Atkins, C. G., Putnam, M. A., and Friebele, E. J., "Instrumentation for interrogating many-element fiber Bragg grating arrays," Proc. SPIE 2444, 257-267 (1995).

[9] Dyer, S.D., Williams, P.A., Espejo, R.J., Kofler, J.D., Etzel, S.M., "Fundamental limits in fiber Bragg grating peak wavelength measurements," (Invited Paper) Proc. SPIE 5855, 88-93 (2005).

[10]Zeh, T., Schweiser, H., Meixner, A.,, et al. "Enhancement of detection accuracy of fiber Bragg grating sensors," 2nd European Workshop on Optical Fibre Sensors, Proc. SPIE 5502, 540-543 (2004).

[11] Lamberti, A.; Vanlanduit, S.; De Pauw, B.; Berghmans, F. "A novel fast phase correlation algorithm for peak wavelength detection of fiber Bragg grating sensors," Opt. Express, 22, 7099-7112 (2014). 